\title{
Distancia recorrida en la prueba de caminata de seis minutos en población adulta sana en una institución de salud de la ciudad de Barranquilla
}

\author{
Distance walked in the six-minute walking \\ test in a healthy adult population at a health \\ care institution in Barranquilla city
}

Lucy Maritza Pérez-Lugo ${ }^{1}$, Jennifer Patricia lobelo-Angulo ${ }^{1}$, Lourdes Varela-Prieto ${ }^{2}$, Carmen

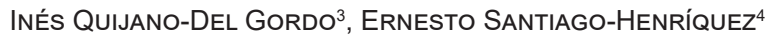

\section{Resumen}

INTRODUCCIÓN: la prueba de caminata en seis minutos (PC6M) es una una prueba de ejercicio submáxima de carga continua no invasiva, reproducible, validada y de bajo costo; en 2002, la American Thoracic Society (ATS) estandarizó la ecuación de Troosters para calcular los valores predichos teniendo en cuenta variables como la edad, la talla y el sexo. Actualmente, en diferentes ciudades de Colombia, estos valores predichos no son aplicables.

MATERIALES Y MÉTODOS: se realizó un estudio descriptivo transversal con 100 pacientes hombres y mujeres sanos entre los 18-80 años, en una institución de salud de la ciudad de Barranquilla, a quienes se les aplicó la PC6M.

RESULTADOS Y DISCUSIÓN: en el grupo total, el promedio de la mejor distancia recorrida fue de 499,8 $\pm 83,6$ metros $(\mathrm{m})(483,1 \pm 61,6 \mathrm{~m}$ en mujeres y 533,7 $\pm 109,7 \mathrm{~m}$ en hombres; $\mathrm{p}<0,05)$, los cuales no guardan relación con los valores obtenidos en ciudades principales de Colombia. En el estudio de Walteros y colaboradores la distancia fue de 658,9 $\mathrm{m}$ en hombres y 592,75 m en mujeres, en Bogotá; en el de Viola y colaboradores fue de 602,7 $\mathrm{m}$ en mujeres y 672,4 m en hombres, también en Bogotá; y en el de Rosero y colaboradores fue de $616 \mathrm{~m}$ en mujeres y $666 \mathrm{~m}$ en hombres, en Cali.

CONCLUSIONES: en la actualidad, las ecuaciones estandarizadas para la PC6M sobrestiman los metros caminados por la población sana entre los 18-80 años, pertenecientes a la institución de salud estudiada de Barranquilla.

Palabras clave: prueba de caminata en 6 minutos, PC6M, Troosters, Barranquilla.

\section{Abstract}

INTRODUCTION: the 6-min walk test (PC6M) is a low-cost, reproducible, validated, non-invasive continuous load submaximal exercise test; the ATS in 2002 standardized the Troosters equation to calculate the predictions taking into account variables such as age, height and sex. Currently in different cities of Colombia, these predictions are not applicable.

MATERIALS AND METHODS: a descriptive cross-sectional study was carried out with 100 healthy male and female patients between $18-80$ years of age in a health institution in the city of Barranquilla to whom the PC6M was applied.

RESULTS AND DISCUSSION: in the total group the average of the best distance traveled was $499.8 \pm 83.6 \mathrm{~m}(483.1 \pm 61.6 \mathrm{~m}$ in women and $533.7 \pm 109.7 \mathrm{~m}$ in men; $p<0.05)$, which are not related to the values obtained in the main cities of Colombia. Walteros et al.
${ }^{1}$ Medicina Interna, Universidad Libre. Barranquilla, Colombia.

${ }^{2}$ Microbiología, magíster en Administración, Metodología de la Investigación. Universidad Libre. Barranquilla, Colombia.

${ }^{3}$ Fisioterapia, Clínica General del Norte, Universidad Libre. Barranquilla, Colombia.

${ }^{4}$ Medicina Interna, Neumología, Clínica General del Norte. Barranquilla, Colombia.

Autor de correspondencia Lucy Maritza Pérez-Lugo.

Correo electrónico:

lucyperezlugo@outlook.com

Recibido: $11 / 05 / 2020$

Aceptado: 23/06/2020 
$658.9 \mathrm{~m}$ in the case of men and $592.75 \mathrm{~m}$ in women in the city of Bogotá; Viola et al. with $602.7 \mathrm{~m}$ in the case of women and 672.4 $\mathrm{m}$ in men also in Bogotá; and Rosero et al. $616 \mathrm{~m}$ in women and $666 \mathrm{~m}$ in men in Cali.

CONCLUSIONS: currently standardized equations for PC6M overestimate the meters walked by the healthy population, between the ages of 18-80 years of the Barranquilla health institution studied.

Keywords: 6-Minute Walk Test; PC6M; Troosters; Barranquilla.

\section{Introducción}

La prueba de caminata en seis minutos (PC6M) se define como una prueba de ejercicio submáxima de carga continua no invasiva, reproducible, validada y de bajo costo (1), la cual es utilizada como predictor de funcionalidad y pronóstico; además, permite evaluar el estado funcional y el umbral ventilatorio del paciente no solo frente a enfermedades respiratorias y cardiovasculares, sino también todas aquellas patologías adicionales que involucren el intercambio gaseoso (2).

La PC6M debe desarrollarse en un terreno plano, nivelado, de 30 metros (m) de largo, sin obstáculos ni circulación de personas. Su fin es identificar la tolerancia del paciente a actividades de la vida diaria, y sirve para evaluar globalmente la respuesta del organismo frente al ejercicio de intensidad leve (3).

Por primera vez en 1999, Troosters realizó la caminata en una universidad belga y propuso una fórmula matemática derivada de ecuaciones de regresión basadas en la edad, el peso y el sexo (4). Si bien esta es la más usada, Enright (5) y Casanova también propusieron ecuaciones matemáticas con las mismas variables, a excepción de Casanova, quien adicionalmente utiliza la frecuencia cardíaca máxima $\left(\mathrm{FC}_{\text {máx }}\right)$ y el \% predicho de la $\mathrm{FC}_{\text {máx }}$ para la estimación de los metros caminados (6). En 2002, la American Thoracic Society (ATS) implementó los estándares, las recomendaciones y los valores predichos que deben usarse para la interpretación de la PC6M, utilizando la ecuación de Troosters (7). Sin embargo, recientemente se ha demostrado que las ecuaciones extranjeras no son adecuadas para poblaciones brasileñas, chilenas, entre otras (8), lo cual no difiere de nuestra población ya que en Colombia las pruebas realizadas en poblaciones de Cali y Bogotá descritas en trabajos publicados por Carvajal y cola- boradores (9), Viola y colaboradores (10) y Walteros y colaboradores (11) no concuerdan con los valores predichos propuestos por Troosters.

Barranquilla no posee estudios de referencia para su población, por lo que se advierte la necesidad de realizar la PC6M con una muestra local de pacientes sanos con el fin de describir, caracterizar y homogeneizar la población adulta sana, establecer valores de distancia recorrida reales, $y$, finalmente, interpretar de manera adecuada la PC6M desde nuestra demografía, etnia y complexión.

\section{Materiales y métodos}

Se realizó un estudio de tipo descriptivo, observacional, transversal con una población de 100 personas sanas que cumplieron con los criterios de inclusión (adultos $>18$ años, hasta los 80 años sin enfermedades osteomusculares y cardiopulmonares asociadas, índice de Charlson $0(12,13)$ y haber firmado de forma voluntaria el consentimiento) y que no cumplan criterios de exclusión (población $<18$ años, antecedentes de enfermedad crónica, presión arterial >180/100 mm Hg, lesiones osteoarticulares que influyen en la capacidad de ejercicio, y no estar de acuerdo con el consentimiento informado), en la Clínica General del Norte desde febrero de 2019 hasta diciembre del mismo año.

Al grupo de 100 personas se les aplicó la PC6M, que consiste en medir la mayor distancia que una persona puede caminar a velocidad constante, sin correr, durante un tiempo continuo de seis minutos (14). La prueba se realizó en un terreno plano, nivelado, de 30 $\mathrm{m}$ de largo, sin obstáculos ni circulación de personas.

Se midieron variables como la escala de Borg (15), la frecuencia cardíaca, la presión arterial y la satura-

Revista Colombiana de Neumología Vol. 32 N. ํ 2 | 2020 
ción de oxígeno al principio y al final de la prueba, así como la frecuencia cardíaca, la saturación de oxígeno y la escala de Borg en los minutos 1 al 6 . Todos los datos se tabularon, pero solo se escogió la mejor prueba de las dos cuando se analizaron los datos.

\section{Resultados}

Al registrar los datos y evidenciar el cumplimiento de los criterios de inclusión, se contó con la participación de 100 personas en la PC6M. El 67 \% de los pacientes fueron mujeres; más del $50 \%$ de participantes tenían edades entre 18-30 años, con un peso entre 60-90 $\mathrm{kg}$. Adicionalmente, el índice de masa corporal (IMC) en el $40 \%$ de los pacientes estuvo entre $18,5-24,9 \mathrm{~kg} /$ $\mathrm{m}^{2}$ (Tabla 1). Del total de los pacientes, solo dos mujeres y un hombre tuvieron que detener la PC6M debido a la dificultad respiratoria que presentaron.

Tabla 1. Distribución porcentual de algunas características biofísicas de los 100 pacientes estudiados en la PC6M

\begin{tabular}{|lcc|}
\hline \multicolumn{1}{|c}{ Variable } & Categoría & $\%$ \\
\hline Sexo & Mujer & 67 \\
& Hombre & 33 \\
& $18-30$ & 54 \\
& $31-50$ & 35 \\
Peso $(\mathrm{kg})$ & $51-80$ & 11 \\
& $<60$ & 19 \\
& $60-90$ & 67 \\
IMC $\left(\mathrm{kg} / \mathrm{m}^{2}\right)$ & $>90$ & 14 \\
& $<18,5$ & 3 \\
& $18,5-24,9$ & 40 \\
& $25-29,9$ & 35 \\
& $30-34,9$ & 16 \\
& $35-39,9$ & 6 \\
\hline Talla & $<150$ & 99 \\
& $150-170$ & 1 \\
\hline
\end{tabular}

Se encontró que las variables de peso, talla e IMC se distribuyen de manera simétrica, a diferencia de las variables edad, distancia recorrida en la primera prueba, segunda prueba y la mejor distancia recorrida, que presentaron una distribución asimétrica. El $50 \%$ de los pacientes tenían una edad a lo sumo de 30 años, y el promedio del IMC fue de $26,4 \pm 4,5$.

El $50 \%$ de los pacientes tuvieron una distancia recorrida en la primera prueba de 466,5 $\mathrm{m}$ (rango intercuartílico $[\mathrm{RIC}]=87,8)$, valor inferior al hallado en la segunda prueba con 492 m $($ RIC $=107,6)$; si al comparar las pruebas se selecciona la mejor distancia, se obtiene una mediana de 497,5 $\mathrm{m}(\mathrm{RIC}=97,5)$. La diferencia de estos valores se debe a que en la primera prueba hubo un valor mínimo de $42 \mathrm{~m}$ y en la segunda prueba un mínimo de $90 \mathrm{~m}$.

En el grupo total, el promedio de la mejor distancia recorrida fue de 499, $8 \pm 83,6 \mathrm{~m}$, siendo $483,1 \pm 61,6 \mathrm{~m}$ en mujeres y $533,7 \pm 109,7 \mathrm{~m}$ en hombres $(p<0,05)$; la diferencia promedio entre las dos pruebas realizadas a cada sujeto fue de 54,1 $\pm 52,8$ en el total de los pacientes, y por género se encontró un promedio superior en hombres que en mujeres: $55,40 \pm 50,8$ y $53,45 \pm 542$, respectivamente (Tabla 2 ).

La distancia recorrida en la primera y la segunda prueba en los hombres difiere de las mujeres $(p<0,05)$ (Tabla 2). Se encontró que la edad no proviene de una población con distribución normal en las categorías de sexo, y al realizar la prueba $U$ de Mann-Whitney se evidenció que la edad de los hombres no difiere de la edad de las mujeres $(p=0,97)$.

Al observar la distribución de los datos entre la mejor distancia según la edad y el sexo, se encontró que, entre los 18-50 años, el sexo femenino recorre una menor distancia; luego, al incrementar la edad entre 5080 , se evidencia que el $50 \%$ de los hombres recorren 270,3 m y como máximo 486 m, a diferencia del $50 \%$ de las mujeres, que recorren 427,5 m y como máximo 483,5 m (Figura 1).

En la Tabla 3 se muestran los cambios presentados en la frecuencia cardíaca y la saturación de oxígeno al inicio y al final de cada una de las pruebas realizadas a los pacientes. En específico, en la primera prueba, la frecuencia cardíaca de los hombres presentó una diferencia de 38,2 latidos por minuto (lpm) a diferencia de la segunda prueba con $37,2 \mathrm{lpm}$. La saturación parcial de oxígeno varió en un $6 \%$ en la primera prueba a un $-1,34 \%$ para la segunda prueba. En cambio, las diferencias en la frecuencia cardíaca en las mujeres pasa- 
ron de 34 lpm a 35 lpm, y las diferencias de saturación parcial de oxígeno para cada prueba fueron de $-0,6 \%$ y $-2,3 \%$, respectivamente.

No existe una correlación significativa entre la mejor distancia recorrida con la edad y las medidas an- tropométricas, a excepción de la talla y el sexo, correlacionadas significativamente en el $43 \%$ y $44 \%$, respectivamente, al nivel de $0,01(p=0,00)$. Sin embargo, al realizar el análisis por sexo no se observaron correlaciones significativas entre la mejor distancia recorrida y las variables antropométricas (Tabla 4).

Tabla 2. Estimación del promedio de las características antropométricas según el sexo en los pacientes estudiados en la PC6M

\begin{tabular}{|lccc|}
\hline Características & Hombres $(\mathrm{DE})$ & Mujeres $(\mathrm{DE})$ & Valor $\boldsymbol{p}$ \\
\hline Talla $(\mathrm{cm})$ & $1,71 \pm 0,08$ & $1,61 \pm 0,07$ & 0,000 \\
\hline Peso $(\mathrm{kg})$ & $82,3 \pm 13,5$ & $66,6 \pm 12,3$ & 0,000 \\
IMC $\left(\mathrm{kg} / \mathrm{m}^{2}\right)$ & $27,9 \pm 3,8$ & $25,6 \pm 4,6$ & 0,013 \\
Distancia P1 $(\mathrm{m})$ & $486,6 \pm 105$ & $446,9 \pm 80,6$ & 0,040 \\
Distancia P2 $(\mathrm{m})$ & $542,2 \pm 79,3$ & $468,5 \pm 82,7$ & 0,000 \\
Mejor distancia $(\mathrm{m})$ & $533,7 \pm 109,7$ & $483,1 \pm 61,6$ & 0,004 \\
Diferencia distancia $(\mathrm{m})$ & $55,40 \pm 50,8$ & $53,45 \pm 542$ & 0,865 \\
\hline
\end{tabular}

cm: centímetros; DE: desviación estándar; IMC: índice de masa corporal; kg: kilogramos. El valor $p<0,05$ se halló mediante la prueba $t$.

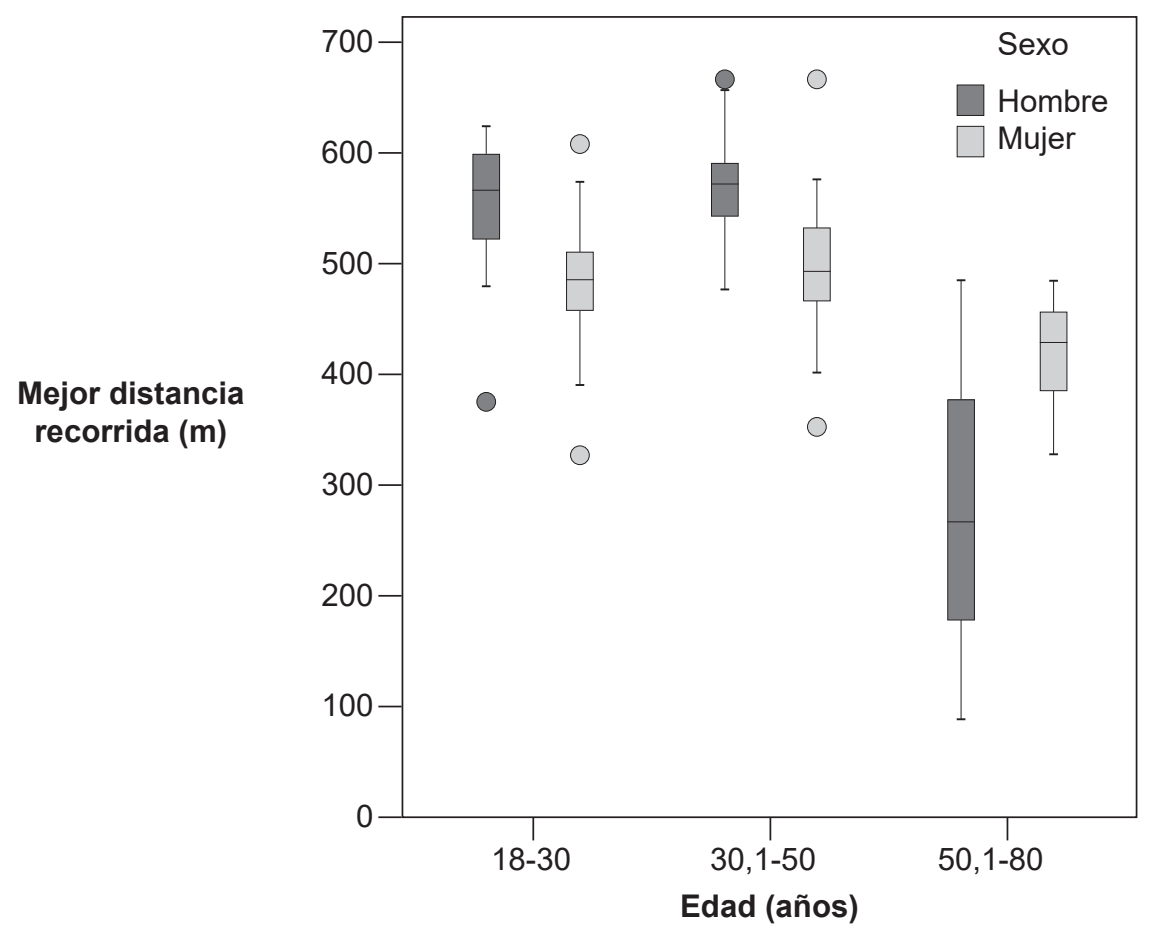

Figura 1. Diagrama de cajas de la mejor distancia recorrida en la PC6M según la edad y el sexo. Los puntos indican los valores extremos. 
Tabla 3. Cambio en la saturación parcial de oxígeno y la frecuencia cardíaca disgregada por sexo en la PC6M

\begin{tabular}{|c|c|c|c|c|c|c|}
\hline \multicolumn{4}{|c|}{ Prueba 1} & \multicolumn{3}{|c|}{ Prueba 2} \\
\hline & Inicial & Final & Diferencia & Inicial & Final & Diferencia \\
\hline Hombres & $\mathrm{DE}$ & & & DE & & \\
\hline FC (Ipm) & $77,7 \pm 10,9$ & $115,8 \pm 17,6$ & $38,1 \pm 16,1$ & $80,3 \pm 12,7$ & $117,6 \pm 22,9$ & $37,3 \pm 21,2$ \\
\hline $\mathrm{SpO}_{2}(\%)$ & $91,8 \pm 2,01$ & $97,8 \pm 1,7$ & $6 \pm 2,1$ & $97,4 \pm 2,4$ & $96,06 \pm 3,4$ & $-1,34 \pm 3,1$ \\
\hline \multicolumn{7}{|l|}{ Mujeres } \\
\hline FC (Ipm) & $79,9 \pm 11,6$ & $113,9 \pm 17,6$ & $34 \pm 16,3$ & $78,1 \pm 12,2$ & $113,1 \pm 19,9$ & $35 \pm 18,5$ \\
\hline $\mathrm{SpO}_{2}(\%)$ & $98,4 \pm 1,2$ & $97,8 \pm 2,4$ & $-0,6 \pm 2,4$ & $97,9 \pm 1,4$ & $95,6 \pm 3,4$ & $-2,3 \pm 2,6$ \\
\hline
\end{tabular}

FC: frecuencia cardíaca; $\mathrm{SpO}_{2}$ : saturación parcial de oxígeno.

Tabla 4. Correlación entre mejor la distancia recorrida, la edad y las medidas antropométricas

\begin{tabular}{|c|c|c|c|c|}
\hline Variables & $\begin{array}{c}\text { Hombres } \\
\text { correlación }\end{array}$ & $\boldsymbol{p}$ & Correlación* & $\begin{array}{c}\text { Mujeres } \\
\boldsymbol{p}\end{array}$ \\
\hline Edad & $-0,064$ & 0,725 & $-0,212$ & 0,085 \\
\hline Talla & 0,224 & 0,211 & 0,221 & 0,073 \\
\hline Peso & $-0,113$ & 0,532 & $-0,027$ & 0,826 \\
\hline IMC & $-0,227$ & 0,204 & $-0,108$ & 0,382 \\
\hline
\end{tabular}

*Correlación de Spearman.

\section{Discusión}

Dentro de los valores obtenidos en el grupo total, el promedio de la mejor distancia recorrida fue de 499,8 $\pm 83,6 \mathrm{~m}(483,1 \pm 61,6 \mathrm{~m}$ en mujeres y $533,7 \pm 109,7$ $\mathrm{m}$ en hombres; $p<0,05)$, los cuales no guardan relación con los valores obtenidos en las ciudades principales de Colombia. En la literatura, en Bogotá, Walteros y colaboradores encontraron $658,9 \mathrm{~m}$ en hombres y $592,75 \mathrm{~m}$ en mujeres; también en Bogotá, Viola y colaboradores con $602,7 \mathrm{~m}$ en mujeres y $672,4 \mathrm{~m}$ en hombres; y en Cali, Rosero y colaboradores con $616 \mathrm{~m}$ en mujeres y $666 \mathrm{~m}$ en hombres.

Un punto importante en nuestra investigación fueron las variables de la prueba, como la edad, el sexo, el peso, el IMC y la talla. Nuestro estudio arrojó datos relevantes, los cuales fueron variables dependientes a la hora de la realización de la prueba. El promedio de edades fue de 30 años y con un IMC de $26,4+-4,5$. Al comparar el promedio entre sexo y variables antropométricas, se encontraron diferencias estadísticamente significativas $(p<0,05)$, siendo mayor en hombres que en mujeres y presentando una correlación directa entre el peso y la talla e inversa con el aumento de la edad, que podrían explicarse por el acondicionamiento físico, la disminución de la fuerza, la masa corporal, las diferencias entre el consumo de oxígeno y el requerimiento menor a medida que se envejece.

En el estudio, la frecuencia cardíaca aumentó para los hombres $(37,3 \pm 10,2$ y $35 \pm 2)$, datos que concuerdan con los estudios publicados por Enright y colaboradores (5), quienes describieron un aumento promedio de $25 \mathrm{lpm}$ en hombres (rango -8 y +79 ) y 20 lpm (rango $-6 \mathrm{y}+58$ ) en las mujeres; esto también tiene relación con los estudios publicados por Rosero y colaboradores (9), con un aumento de 35,4 $\pm 22,8$ en hom- 
bres y $36,7 \pm 18,1$ en mujeres. Adicionalmente, esta variable no se correlacionó con los metros caminados.

Al comparar los resultados con las ecuaciones más utilizadas, Troosters (4), Casanova (6) y Enright (5), los resultados difieren; con Troosters, el promedio a caminar según la edad y el sexo fue de $818,8 \mathrm{~m}$, con una diferencia de promedios $-321 \mathrm{~m}$ de nuestro estudio, lo que significa que la ecuación subvalora nuestros resultados, es decir, los pacientes estudiados caminan menos metros que lo que predice la ecuación. Por otro lado, comparando la ecuación de Enright (5), con la cual la media según la edad y el sexo fue de 630,2 m, la diferencia de promedios de nuestro estudio fue de $-133,3$ m. Finalmente, con respecto a la ecuación planteada por Casanova (6), los promedios de los valores predichos según la edad, el sexo, la frecuencia cardíaca máxima y el \% predicho de la frecuencia cardíaca máxima, se presentó una diferencia de $+58 \mathrm{~m}$, por lo que fue la ecuación con mayor aproximación a la población estudiada.

\section{Conclusiones}

En la actualidad, las ecuaciones estandarizadas para la PC6M (Troosters, Casanova y Enright) sobrestiman los metros caminados para el caso de la población sana estudiada entre las edades de 18-80 años de la institución de salud de Barranquilla. La ecuación de Casanova fue la que mejor aproximación presentó en comparación con el resto de las ecuaciones.

Debido a que los resultados obtenidos no son suficientes para determinar los valores predichos normales para la PC6M, para futuras investigaciones recomendamos la continuidad del estudio para obtener una muestra significativa que represente a la población barranquillera, estandarizando cuánto es el promedio caminado según la complexión, la edad, la etnia y otras características antropométricas.

\section{Financiación}

Los autores han declarado que no se recibió apoyo financiero de ninguna organización para este trabajo.

\section{Referencias}

1. Tokmakova M. Cardiopulmonary stress test--general characteristics and clinical value. Folia Med (Plovdiv). 1998;40(3B Suppl 3):38-42.

2. Ross RM, Murthy JN, Wollak ID, Jackson AS. The six minute walk test accurately estimates mean peak oxygen uptake. BMC Pulm Med. 2010;10:31. doi:10.1186/1471-2466-10-31

3. Stoller, J. K. (2015). Murray \& Nadel's Textbook of Respiratory Medicine, 6th Edition. Annals of the American Thoracic Society. 2015;12(8):1257-8. doi:10.1513/annalsats.201504-251ot

4. Troosters T, Gosselink R, Decramer M. Six minute walking distance in healthy elderly subjects. Eur Respir J. 1999;14(2):270-274. doi:10.1034/j.1399-3003.1999.14b06.x

5. Enright PL, Sherrill DL. Reference equations for the six-minute walk in healthy adults [published correction appears in Am J Respir Crit Care Med. 2020 Feb 1;201(3):393]. Am J Respir Crit Care Med. 1998;158(5Pt1):1384-87. doi:10.1164/ ajrccm.158.5.9710086

6. Casanova C, Celli BR, Barria P, et al. The 6-min walk distance in healthy subjects: reference standards from seven countries. Eur Respir J. 2011;37(1):150-156. doi:10.1183/09031936.00194909

7. ATS Committee on Proficiency Standards for Clinical Pulmonary Function Laboratories. ATS statement: guidelines for the six-minute walk test [published correction appears in Am J Respir Crit Care Med. 2016 May 15;193(10):1185]. Am J Respir Crit Care Med. 2002;166(1):111-117. doi:10.1164/ ajrccm.166.1.at1102

8. Dourado VZ. Equações de Referência para o Teste de Caminhada de Seis Minutos em Indivíduos Saudáveis [Reference Equations for the 6-Minute Walk Test in Healthy Individuals. Arq Bras Cardiol. 2011;S0066-782X2011005000024.

9. Rosero-Carvajal HE, Cuero-Campáz DF, Arias-Balanta AJ, Wilches-Luna EC. Distancia recorrida en la prueba de marcha de los 6 minutos en una población caleña sana de entre 20 y 65 años. Estudio piloto. Rehabilitación. 2017;51(3):143-8. doi:10.1016/j.rh.2016.12.003

10. Viola-Muñoz LC, Casas A, Gómez V, Aguiar P, Sacristan L, Maldonado D. Valores normales de referencia de la prueba de marcha de seis minutos (P6M) en adultos sanos mayores de 18 años en Bogotá, Colombia. Univ la Sabana. 2015. Disponible en: https://intellectum.unisabana.edu.co/handle/10818/17826

11. Walteros-Manrique RE. Distancia recorrida de la prueba de caminata de seis minutos en población adulta sana en una comunidad universitaria de la ciudad de Bogotá [Tesis especialidad]. Bogotá: Universidad Nacional de Colombia; 2018. Disponible en: http://bdigital.unal.edu.co/65066/2/RaulWalteros.2018.pdf

12. Charlson ME, Pompei P, Ales KL, MacKenzie CR. A new method of classifying prognostic comorbidity in longitu- 
dinal studies: development and validation. J Chronic Dis. 1987;40(5):373-383. doi:10.1016/0021-9681(87)90171-8

13. Quan H, Li B, Couris CM, Fushimi K, Graham P, Hider P, et al. Updating and validating the Charlson comorbidity index and score for risk adjustment in hospital discharge abstracts using data from 6 countries. Am J Epidemiol. 2011;173(6):676-682. doi:10.1093/aje/kwq433
14. González NF, Anchique C V, Rivas AD. Test de caminata de 6 minutos en pacientes de rehabilitación cardiaca de altitud moderada. Rev Colomb Cardiol. 2017;24(6):626-32.

15. Borg GA. Psychophysical bases of perceived exertion. Med Sci Sports Exerc. 1982;14(5):377-81. 